Internat. J. Math. \& Math. Sci.

Vol. 24, No. 11 (2000) 773-779

S0161171200004427

(C) Hindawi Publishing Corp.

\title{
COMMON STATIONARY POINTS OF MULTIVALUED MAPPINGS ON BOUNDED METRIC SPACES
}

\author{
ZEQING LIU and SHIN MIN KANG
}

(Received 31 August 1999 and in revised form 17 March 2000)

\begin{abstract}
Necessary and sufficient conditions for the existence of common stationary points of two multivalued mappings and common stationary point theorems for multivalued mappings on bounded metric spaces are given. Our results extend the theorems due to Fisher in 1979, 1980, and 1983 and Ohta and Nikaido in 1994.
\end{abstract}

Keywords and phrases. Common stationary point, multivalued mappings, complete bounded metric space.

2000 Mathematics Subject Classification. Primary 54H25.

1. Introduction. Let $(X, d)$ be a metric space and $B(X)$ denote the set of all nonempty bounded subsets of $X$. For $A, B \in X$, let $\delta(A, B)=\sup \{d(a, b): a \in A, b \in B\}$ and $\delta(A)=\delta(A, A)$. If $A$ consists of a single point $a$, we write $\delta(A, B)=\delta(a, B)$. If $B$ also consists of a single point $b$, we write $\delta(A, B)=\delta(a, b)=d(a, b)$. Let $N$ and $\omega$ denote the sets of positive integers and nonnegative integers, respectively. Let $\Phi$ denote a family of mappings such that for each $\phi \in \Phi, \phi:[0, \infty) \rightarrow[0, \infty)$ is upper semicontinuous, nondecreasing and $\phi(t)<t$ for $t>0$.

The following definitions and lemmas were introduced by Fisher [3] and Singh and Meade [6].

DeFinITION 1.1 [3]. Let $\left\{A_{n}\right\}$ be a sequence of sets in $B(X)$ and $A \in B(X)$. The sequence $\left\{A_{n}\right\}$ is said to converge to the set $A$ if

(i) each point $a \in A$ is the limit of some convergent sequence $\left\{a_{n}\right\}$, where $a_{n} \in A_{n}$ for $n \in N$;

(ii) for arbitrary $\epsilon>0$, there exists $k \in N$ such that $A_{n} \subseteq A_{\epsilon}$, for $n>k$, where $A_{\epsilon}$ is the union of all open spheres with centres in $A$ and radius $\epsilon$.

DefinITION 1.2 [3]. Let $F$ be a multivalued mapping of $(X, d)$ into $B(X)$. The mapping $F$ is called continuous in $X$ if whenever $\left\{x_{n}\right\}$ is a sequence of points in $X$ converging to $x \in X$, the sequence $\left\{F x_{n}\right\}$ in $B(X)$ converges to $F x \in B(X)$.

LEMMA 1.3 [3]. If $\left\{A_{n}\right\}$ and $\left\{B_{n}\right\}$ are sequences of bounded subsets of a complete metric space $(X, d)$ which converge to the bounded subsets $A$ and $B$, respectively, then the sequence $\left\{\delta\left(A_{n}, B_{n}\right)\right\}$ converges to $\delta(A, B)$.

LEMMA 1.4 [6]. Let $\phi \in \Phi$. Then $\phi(t)<t$ for each $t>0$ if and only if

$$
\lim _{n \rightarrow \infty} \phi^{n}(t)=0,
$$

where $\phi^{n}$ denotes the $n$-times composition of $\phi$. 
Let $F$ and $G$ be mappings of $(X, d)$ into $B(X)$. A point $x \in X$ is called a common stationary point of $F$ and $G$ if $F x=G x=\{x\}$. For $A \subseteq X$, let $F A=\cup_{a \in A} F a$ and $G F A=G(F A)$. The mappings $F$ and $G$ are said to commute if $F G x=G F x$ for $x \in X$. Define $C_{F}=\{T: T$ is a mapping of $X$ into $B(X)$ and $T$ and $F$ commute $\}$ and $C C_{F}=\left\{T: T\right.$ is continuous and $\left.T \in C_{F}\right\}$. It follows that $C_{F} \supseteq\left\{F^{n}: n \in \omega\right\}$, where $F^{0} x=\{x\}$ for $x \in X$.

Throughout the rest of the paper, we assume that $(X, d)$ is a complete bounded metric space.

In 1979, Fisher [1] proved a common fixed point theorem for commuting mappings $f$ and $g$ of $(X, d)$ into itself satisfying

$$
d(f x, g y) \leq c \max \{d(x, y), d(x, f x), d(y, g y), d(x, g y), d(y, f x)\}
$$

for all $x, y \in X$, where $0 \leq c<1$.

In 1980, Fisher [2] generalized the result to multivalued mappings $F$ and $G$ of $(X, d)$ into $B(X)$ satisfying the condition

$$
\delta(F x, G y) \leq c \max \{\delta(x, y), \delta(x, F x), \delta(y, G y), \delta(x, G y), \delta(y, F x)\}
$$

for all $x, y \in X$, where $0 \leq c<1$.

In 1983, Fisher [4] established a common fixed point theorem for continuous, commuting mappings $F$ and $G$ of $(X, d)$ into $B(X)$ satisfying

$$
\begin{array}{r}
\delta\left(F^{p} G^{p} x, F^{p} G^{p} y\right) \leq c \max \{ \\
\delta\left(F^{q} G^{r} x, F^{s} G^{t} y\right), \delta\left(F^{q} G^{r} x, F^{s} G^{t} x\right), \\
\left.\delta\left(F^{q} G^{r} y, F^{s} G^{t} y\right): 0 \leq q, r, s, t \leq p\right\}
\end{array}
$$

for all $x, y \in X$, where $0 \leq c<1$ and $p$ is a fixed positive integer.

In 1994, Ohta and Nikaido [5] obtained the existence of fixed point for a continuous self mapping $f$ of $(X, d)$ satisfying

$$
d\left(f^{k} x, f^{k} y\right) \leq c \delta\left(\left\{f^{i} t: t \in\{x, y\}, i \in \omega\right\}\right)
$$

for all $x, y \in X$, where $0 \leq c<1$ and $k$ is a fixed positive integer.

The first purpose of the paper is to establish criteria for the existence of common stationary points of commuting mappings $F$ and $G$ of $(X, d)$ into $B(X)$. The second purpose of the paper is to prove common stationary point theorems for commuting mappings $F$ and $G$ of $(X, d)$ into $B(X)$ satisfying one of the following:

$$
\delta\left(F^{p} G^{p} x, F^{q} G^{q} y\right) \leq \phi\left(\delta\left(\cup_{D \in C_{F G}} D\{x, y\}\right)\right)
$$

for all $x, y \in X$, where $\phi \in \Phi$ and $p, q$ are fixed positive integers;

$$
\delta\left(F^{p} x, G^{q} y\right) \leq \phi\left(\delta\left(\cup_{D \in C_{F} \cap C_{G}} D\{x, y\}\right)\right)
$$

for all $x, y \in X$, where $\phi \in \Phi$ and $p, q$ are fixed positive integers;

$$
\delta(F x, G y) \leq \phi\left(\max \left\{\delta(x, F x), \delta(y, G y), \delta(x, G y), \delta(y, F x), \delta\left(\cup_{D \in C C_{F G}} D\{x, y\}\right)\right\}\right)
$$

for all $x, y \in X$, where $\phi \in \Phi$. 
It is easy to see that (1.2) and (1.3) are special cases of (1.8), that (1.4) and (1.5) are special cases of (1.6), and that (1.2) and (1.5) are special cases of (1.7). Our results extend and unify the theorems of Fisher [1, 2, 4] and Ohta and Nikaido [5].

2. Common stationary points. Our main results are as follows.

THEOREM 2.1. Let $F$ and $G$ be continuous and commuting mappings of $(X, d)$ into $B(X)$. Then the following statements are equivalent:

(i) $F$ and $G$ have a common stationary point;

(ii) there exist $S, T \in C C_{F} \cap C C_{G}$ with $S \in C_{T}$ and $\phi \in \Phi$ such that

$$
\delta(S x, T y) \leq \phi\left(\delta\left(\cup_{D \in C C_{S} \cap C C_{T}} D\{x, y\}\right)\right) \quad \forall x, y \in X ;
$$

(iii) there exist $S, T \in C_{F} \cap C_{G}$ with $S \in C_{T}$ and $\phi \in \Phi$ such that

$$
\delta(S x, T y) \leq \phi\left(\delta\left(\cup_{D \in C_{S} \cap C_{T}} D\{x, y\}\right)\right) \quad \forall x, y \in X ;
$$

(iv) there exist mappings $S, T$ of $(X, d)$ into $B(X)$ with $S \in C_{T}$ and $\phi \in \Phi$ such that $F, G \in C_{S T}$ and

$$
\delta(S x, T y) \leq \phi\left(\delta\left(\cup_{D \in C_{S T}} D\{x, y\}\right)\right) \quad \forall x, y \in X .
$$

Proof. We shall verify the following implications: (i) $\Rightarrow($ ii $) \Rightarrow($ iii) $\Rightarrow($ iv $) \Rightarrow($ i). Suppose, first of all, that $F$ and $G$ have a common stationary point $z$. Define mappings $S$ and $T$ of $(X, d)$ into $B(X)$ by $S x=T x=\{z\}$ for all $x \in X$. It is easy to check that $S, T \in$ $C C_{F} \cap C C_{G}$ and

$$
\delta(S x, T y)=0 \leq \phi\left(\delta\left(\cup_{D \in C C_{S} \cap C C_{T}} D\{x, y\}\right)\right)
$$

for all $x, y \in X, \phi \in \Phi$, that is, (ii) holds.

Note that $C C_{F} \subseteq C_{F}$ and $C_{S} \cap C_{T} \subseteq C_{S T}$. Therefore (ii) $\Rightarrow$ (iii) $\Rightarrow$ (iv) are clear.

We now assume that (iv) holds. Then for any $A, B \in B(X)$, we have

$$
\delta(S A, T B) \leq \phi\left(\delta\left(\cup_{D \in C_{S T}} D(A \cup B)\right)\right),
$$

by (iv). Since $X$ is bounded, $M=\delta(X)<\infty$. Set $X_{n}=S^{n} T^{n} X$ for $n \in N$. Then $X_{n} \subseteq X_{n-1}$ for $n \in N$. We now will prove by induction that

$$
\delta\left(X_{n}\right) \leq \phi^{n}(M) \text { for } n \in N .
$$

Note that $S$ and $T$ commute. From (2.5), we have

$$
\delta\left(X_{1}\right)=\delta(S T X, T S X) \leq \phi\left(\delta\left(\cup_{D \in C_{S T}} D(T X \cup S X)\right)\right) \leq \phi(\delta(X))=\phi(M),
$$

that is, (2.6) holds for $n=1$. Assume now that (2.6) holds for some $n \in N$. It follows from (2.5) that

$$
\begin{aligned}
\delta\left(X_{n+1}\right) & =\delta\left(S^{n+1} T^{n+1} X, T^{n+1} S^{n+1} X\right) \\
& \leq \phi\left(\delta\left(\cup_{D \in C_{S T}} D\left(S^{n} T^{n+1} X \cup T^{n} S^{n+1} X\right)\right)\right) \\
& =\phi\left(\delta\left(\cup_{D \in C_{S T}} S^{n} T^{n}(D T X \cup D S X)\right)\right) \\
& \leq \phi\left(\delta\left(X_{n}\right)\right) \leq \phi^{n+1}(M)
\end{aligned}
$$


by our assumption. Hence (2.6) follows by induction. Choose $x_{n} \in X_{n}$ for $n \in N$. Then, by (2.6), we get

$$
d\left(x_{n}, x_{m}\right) \leq \delta\left(X_{n}, X_{m}\right) \leq \delta\left(X_{n}\right) \leq \phi^{n}(M) \text { for } m>n .
$$

Consequently, $\left\{x_{n}\right\}$ is a Cauchy sequence by Lemma 1.4. Since $X$ is complete, there exists a point $z$ in $X$ such that $x_{n} \rightarrow z$ as $n \rightarrow \infty$. From (2.6), we have

$$
\delta\left(z, X_{n}\right) \leq d\left(z, x_{m}\right)+\delta\left(x_{m}, X_{n}\right) \leq d\left(z, x_{m}\right)+\delta\left(X_{n}\right) \leq d\left(z, x_{m}\right)+\phi^{n}(M)
$$

for $m, n \in N$ with $m>n$. Letting $m$ tend to infinity, we obtain

$$
\delta\left(z, X_{n}\right) \leq \phi^{n}(M) \text { for } n \in N .
$$

Since $F$ is continuous and $x_{n} \rightarrow z$, then $\left\{F x_{n}\right\}$ converges to $\{F z\}$. Note that

$$
F x_{n} \subseteq F S^{n} T^{n} X=S^{n} T^{n} F X \subseteq X_{n} \quad \text { for } n \in N .
$$

Then $\delta\left(z, F x_{n}\right) \leq \delta\left(z, X_{n}\right)$ for $n \in N$. Letting $n$ tend to infinity, we have $\delta(z, F z) \leq 0$ by (2.11) and Lemmas 1.3 and 1.4 , that is, $F z=\{z\}$. Similarly, we have $G z=\{z\}$. This completes the proof.

THEOREM 2.2. Let $F$ and $G$ be continuous and commuting mappings of $(X, d)$ into $B(X)$ satisfying (1.6) or (1.7). Then $F$ and $G$ have a unique common stationary point $z$ and the sequence $\left\{F^{n} G^{n} x\right\}$ converges to $\{z\}$ for all $x \in X$.

Proof. Let $M=\delta(X), k=p+q, X_{n}=F^{n} G^{n} X$ and $x_{n} \in X_{n}$ for $n \in N$. Note that every $n \in N$ can be written as

$$
n=k j+i,
$$

where $j \in \omega$ and $0 \leq i<k$. Now we claim that

$$
\delta\left(X_{n}\right) \leq \phi^{j}(M) .
$$

If (1.6) is satisfied, then

$$
\begin{aligned}
\delta\left(X_{n}\right) & =\delta\left(F^{p} G^{p}\left(F^{q+i} G^{q+i} X_{k(j-1)}\right), F^{q} G^{q}\left(F^{p+i} G^{p+i} X_{k(j-1)}\right)\right) \\
& \leq \phi\left(\delta\left(\cup_{D \in C_{F G}} D\left(F^{q+i} G^{q+i} X_{k(j-1)} \cup F^{p+i} G^{p+i} X_{k(j-1)}\right)\right)\right) \\
& =\phi\left(\delta\left(\cup_{D \in C_{F G}}\left(F^{k(j-1)} G^{k(j-1)} F^{q+i} G^{q+i} D X \cup F^{k(j-1)} G^{k(j-1)} F^{p+i} G^{p+i} D X\right)\right)\right) \\
& \leq \phi\left(\delta\left(X_{k(j-1)}\right)\right)
\end{aligned}
$$

which implies that

$$
\delta\left(X_{k j}\right) \leq \phi\left(\delta\left(X_{k(j-1)}\right)\right) \leq \cdots \leq \phi^{j-1}\left(\delta\left(X_{k}\right)\right) \leq \phi^{j}(M) .
$$

Note that $X_{n} \subseteq X_{n-1}$. Thus (2.14) follows from (2.15) and (2.16). If (1.7) is satisfied, then

$$
\begin{aligned}
\delta\left(X_{n}\right) & =\delta\left(F^{p}\left(F^{q+i} G^{k+i} X_{k(j-1)}\right), G^{q}\left(F^{k+i} G^{p+i} X_{k(j-1)}\right)\right) \\
& \leq \phi\left(\delta\left(\cup_{D \in C_{F} \cap C_{G}} D\left(F^{q+i} G^{k+i} X_{k(j-1)} \cup F^{k+i} G^{p+i} X_{k(j-1)}\right)\right)\right) \\
& \leq \phi\left(\delta\left(X_{k(j-1)}\right)\right) .
\end{aligned}
$$


Similarly, (2.16) holds also. It follows from (2.16) and (2.17) that (2.14) holds.

Given $x_{n} \in X_{n}$ for all $n \in N$. For any $m>n>k$, by (2.13) and (2.14) we have

$$
d\left(x_{n}, x_{m}\right) \leq \delta\left(X_{n}\right) \leq \phi^{j}(M) .
$$

As in the proof of Theorem 2.1, we conclude that $F$ and $G$ have a common stationary point $z$ and that $x_{n} \rightarrow z$ as $n \rightarrow \infty$. Suppose that $F$ and $G$ have a second common stationary point $w$. Then $\{u\}=F^{n} G^{n} u \subseteq X_{n}$ for $u \in\{z, w\}$ and $n \in N$. In view of (2.13) and (2.14), we infer that

$$
d(z, w) \leq \delta\left(X_{n}\right) \leq \phi^{j}(M) .
$$

Letting $n$ tend to infinity we have $d(z, w) \leq 0$ by Lemma 1.4 , that is, $z=w$. Hence $F$ and $G$ have a unique common stationary point $z$. For $x \in X$ and $n \in N$, choose $y_{n} \in F^{n} G^{n} x$. Using (2.13) and (2.14), we have

$$
d\left(y_{n}, z\right) \leq \delta\left(F^{n} G^{n} x, z\right) \leq \delta\left(X_{n}, z\right) \leq \delta\left(X_{n}\right) \leq \phi^{j}(M) .
$$

Letting $n$ tend to infinity, by Lemma 1.4 and Definition 1.1 and the above inequalities, we conclude that $\left\{F^{n} G^{n} x\right\}$ converges to $\{z\}$. This completes the proof.

As a consequence of Theorem 2.2, we have the following corollary.

COROLLARY 2.3. Let $F$ and $G$ be continuous and commuting mappings of $(X, d)$ into $B(X)$ satisfying one of the following:

$$
\delta\left(F^{q} x, G^{q} y\right) \leq \phi\left(\delta\left(\cup_{i, j \in \omega} F^{i} G^{j}\{x, y\}\right)\right) \quad \forall x, y \in X,
$$

where $\phi \in \Phi$ and $p, q$ are fixed positive integers;

$$
\delta\left(F^{p} G^{p} x, F^{q} G^{q} y\right) \leq \phi\left(\delta\left(\cup_{i, j \in \omega} F^{i} G^{j}\{x, y\}\right)\right) \quad \forall x, y \in X,
$$

where $\phi \in \Phi$ and $p, q$ are fixed positive integers. Then $F$ and $G$ have a unique common stationary point $z$ and the sequence $\left\{F^{n} G^{n} x\right\}$ converges to $\{z\}$ for all $x \in X$.

From Corollary 2.3, we have the following.

COROLlary 2.4 [4, Theorem 1]. Let $F$ and $G$ be continuous and commuting mappings of $(X, d)$ into $B(X)$ satisfying (1.3). Then $F$ and $G$ have a unique common stationary point $z$ and the sequence $\left\{F^{n} G^{n} x\right\}$ converges to $\{z\}$ for all $x \in X$.

COROLlary 2.5 [5, Theorem 3]. Let $f$ be a continuous mapping of $(X, d)$ into itself satisfying (1.5). Then $f$ has a unique fixed point $z$ and for each $x \in X, f^{n} x \rightarrow z$ as $n \rightarrow \infty$.

THEOREM 2.6. Let $F$ and $G$ be commuting mappings of $(X, d)$ into $B(X)$ satisfying (1.8). Then $F$ and $G$ have a unique common stationary point $z$ and the sequence $\left\{F^{n} G^{n} x\right\}$ converges to $\{z\}$ for all $x \in X$. Further, $\{z\}=D z$ for all $D \in C C_{F G}$.

Proof. Let $M=\delta(X), X_{n}=F^{n} G^{n} X$, and $x_{n} \in X_{n}$ for $n \in N$. As in the proof of Theorem 2.1, we conclude that $\delta\left(X_{n}\right) \leq \phi^{n}(M)$ for $n \in N$ and that $x_{n} \rightarrow z, \delta\left(z, X_{n}\right) \rightarrow$ 
0 as $n \rightarrow \infty$. Consequently, the sequences $\left\{X_{n}\right\}$ and $\left\{\{z\} \cup X_{n}\right\}$ converge to $\{z\}$. For each $D \in C C_{F G}$, we have $\delta\left(D x_{n}, z\right) \rightarrow \delta(D z, z)$ as $n \rightarrow \infty$, by the continuity of $D$ and Lemma 1.3. Note that

$$
\begin{aligned}
\delta\left(D x_{n}, z\right) & \leq \delta\left(D x_{n}, x_{n}\right)+d\left(x_{n}, z\right) \leq \delta\left(X_{n}\right)+d\left(x_{n}, z\right) \\
& \leq \phi^{n}(M)+d\left(x_{n}, z\right) \longrightarrow 0 \text { as } n \rightarrow \infty,
\end{aligned}
$$

which implies that $\delta(D z, z) \leq 0$, that is, $D z=\{z\}$. Using (1.8), we have for $n \in N$,

$$
\begin{aligned}
& \delta\left(F^{n} G^{n} X, G z\right) \\
& \leq \phi\left(\operatorname { m a x } \left\{\delta\left(F^{n-1} G^{n} X, F^{n} G^{n} X\right), \delta(z, G z), \delta\left(F^{n-1} G^{n} X, G z\right),\right.\right. \\
& \left.\left.\delta\left(z, F^{n} G^{n} X\right), \delta\left(\cup_{D \in C C_{F G}} D\left(F^{n-1} G^{n} X \cup\{z\}\right)\right)\right\}\right) \\
& \leq \phi\left(\operatorname { m a x } \left\{\delta\left(X_{n-1}, X_{n}\right), \delta(z, G z), \delta\left(X_{n-1}, G z\right)\right.\right. \text {, } \\
& \left.\left.\delta\left(z, X_{n}\right), \delta\left(\cup_{D \in C C_{F G}}\left(F^{n-1} G^{n-1} D G X \cup D z\right)\right)\right\}\right) \\
& \leq \phi\left(\operatorname { m a x } \left\{\delta\left(X_{n-1}\right), \delta(z, G z), \delta\left(X_{n-1}, G z\right)\right.\right. \text {, } \\
& \left.\left.\delta\left(z, X_{n}\right), \delta\left(X_{n-1} \cup\{z\}\right)\right\}\right),
\end{aligned}
$$

which implies that

$$
\begin{aligned}
\delta\left(x_{n}, G z\right) & \leq \delta\left(F^{n} G^{n} X, G z\right) \\
& \leq \phi\left(\max \left\{\delta(z, G z), \delta\left(X_{n-1}, G z\right), \delta\left(z, X_{n}\right), \delta\left(X_{n-1} \cup\{z\}\right)\right\}\right) .
\end{aligned}
$$

Letting $n$ tend to infinity, we get

$$
\delta(z, G z) \leq \phi(\max \{\delta(z, G z), \delta(z, G z), 0,0\})=\phi(\delta(z, G z)) .
$$

Suppose that $\delta(z, G z)>0$. Then

$$
\delta(z, G z) \leq \phi(\delta(z, G z))<\delta(z, G z)
$$

which is a contradiction. Therefore $\delta(z, G z)=0$, that is, $G z=\{z\}$. Similarly we have $F z=\{z\}$. The rest of the proof is exactly the same as that of Theorem 2.2. This completes the proof.

From Theorem 2.6, we have the following corollary.

COROLlary 2.7 [2, Theorem 2]. Let $F$ and $G$ be commuting mappings of $(X, d)$ into $B(X)$ satisfying (1.3). Then $F$ and $G$ have a unique common stationary point $z$ and the sequence $\left\{F^{n} G^{n} x\right\}$ converges to $\{z\}$ for all $x \in X$.

COROLlaRY 2.8 [1, Theorem 4]. Let $f$ and $g$ be commuting mappings of $(X, d)$ into itself satisfying (1.2). Then $f$ and $g$ have a unique common fixed point $z$ and for each $x \in X, f^{n} g^{n} \rightarrow z$ as $n \rightarrow \infty$.

The following example shows that Theorem 2.6 extends properly Corollaries 2.7 and 2.8. 
EXAMPLE 2.9. Let $X=\{1,2,5,8\}$ with the usual metric. Define self mappings $f$ and $g$ of $(X, d)$ by

$$
f 1=1, \quad f 2=f 5=g 1=g 2=g 5=5, \quad f 8=g 8=2 .
$$

Set $F x=\{f x\}$ and $G x=\{g x\}$ for $x \in X$. Let $\phi(t)=(1 / 2) t$ for $t \geq 0$. It is easy to check that $F$ and $G$ satisfy the conditions of Theorem 2.6. But Corollaries 2.7 and 2.8 are not applicable since

$$
d(f 1, g 1)=4=\max \{d(1,1), d(1, f 1), d(1, g 1), d(1, g 1), d(1, f 1)\},
$$

that is, $f$ and $g$ do not satisfy (1.2). Similarly $F$ and $G$ do not satisfy (1.3).

ACKNOWLEDGEMENT. The authors would like to thank the referee for his many helpful comments. The second author was supported by Korea Research Foundation Grant (KRF-99-005-D00003).

\section{REFERENCES}

[1] B. Fisher, Results on common fixed points on bounded metric spaces, Math. Sem. Notes Kobe Univ. 7 (1979), no. 1, 73-80. MR 80h:54057. Zbl 415.54029.

[2] _ Set-valued mappings on bounded metric spaces, Indian J. Pure Appl. Math. 11 (1980), no. 1, 8-12. MR 83b:54058. Zbl 421.54030.

[3] C Common fixed points of mappings and set-valued mappings, Rostock. Math. Kolloq. (1981), no. 18, 69-77. MR 83e:54041. Zbl 479.54025.

[4] _ Common fixed points of set-valued mappings on bounded metric spaces, Math. Sem. Notes Kobe Univ. 11 (1983), no. 2, part 2, 307-311. MR 86i:54043. Zbl 579.54032.

[5] M. Ohta and G. Nikaido, Remarks on fixed point theorems in complete metric spaces, Math. Japon. 39 (1994), no. 2, 287-290. MR 94m:54099. Zbl 802.47055.

[6] S. P. Singh and B. A. Meade, On common fixed point theorems, Bull. Austral. Math. Soc. 16 (1977), no. 1, 49-53. MR 55\#11234. Zbl 351.54040.

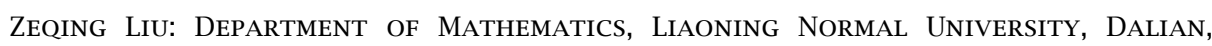
LIAONING 116029, CHINA

Shin Min Kang: Department of Mathematics, Gyeongsang National University, CHINJU 660-701, KOREA

E-mail address: smkang@nongae.gsnu.ac. kr 


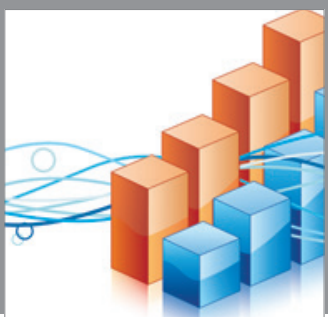

Advances in

Operations Research

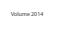

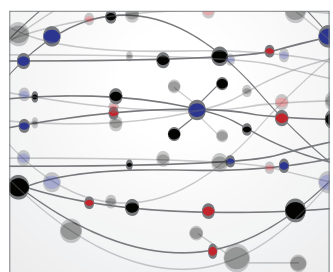

\section{The Scientific} World Journal
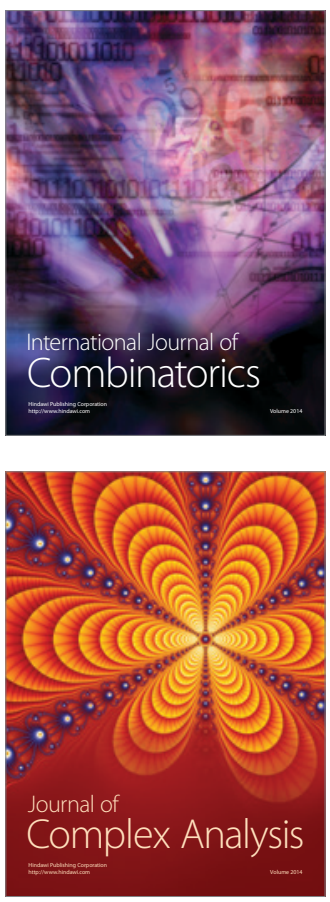

International Journal of

Mathematics and

Mathematical

Sciences
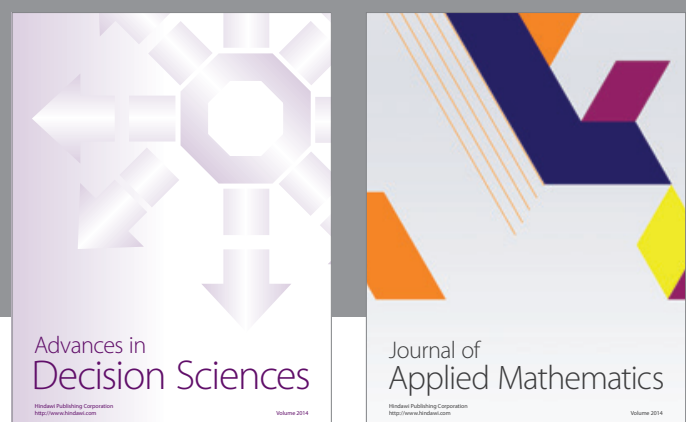

Journal of

Applied Mathematics
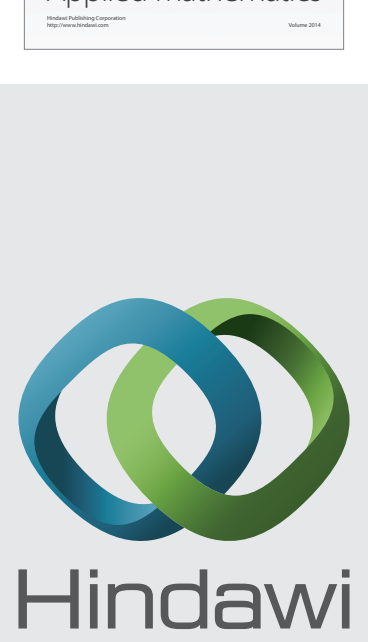

Submit your manuscripts at http://www.hindawi.com
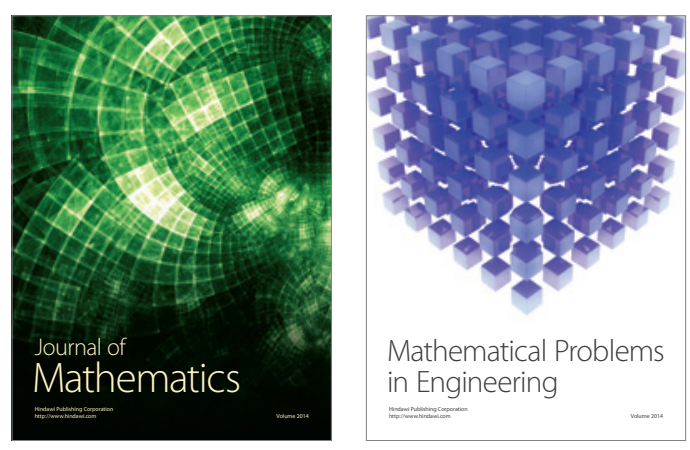

Mathematical Problems in Engineering
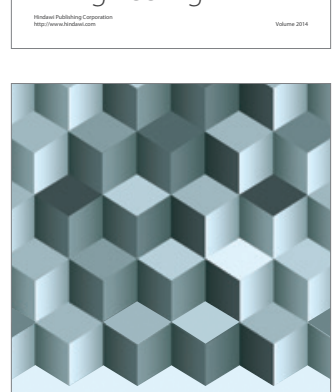

Journal of

Function Spaces
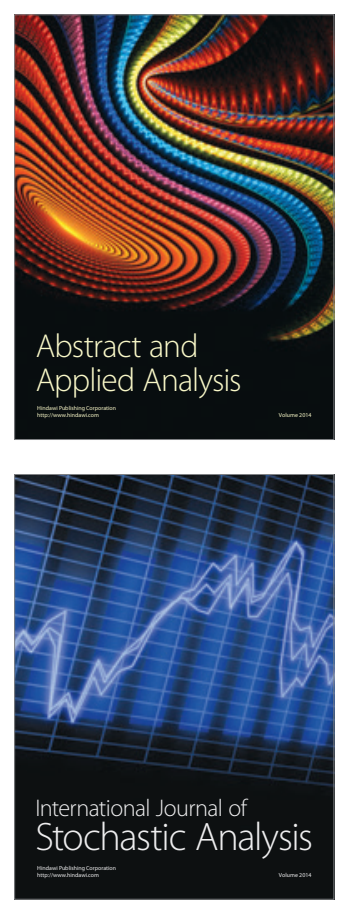

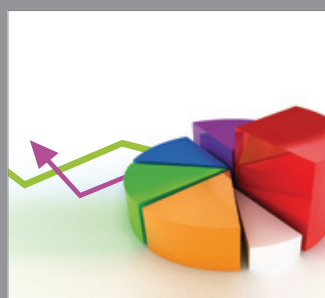

ournal of

Probability and Statistics

Promensencen
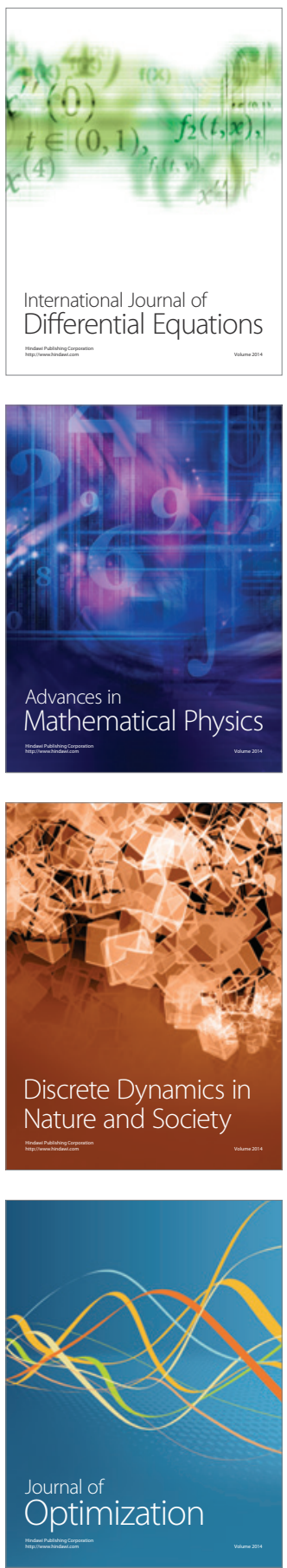